\title{
Forage production of nine alfalfa cultivars in the semiarid region of Puerto Rico'
}

\author{
Luis E. Tergas, Jaime Vélez-Santiago and Doralisa Vera de Saldaña ${ }^{2}$
}

\begin{abstract}
Dry forage production of alfalfa (Medicago sativa) cultivars with overhead irrigation was determined by cutting every 30 to 35 days for 3 years (1983-85) af the Fortuna Agriculfural Research and Development Center. Florida 66 and 77, and a mixture of these two cultivars, produced highest yields with mean annual dry forage (DF) of $25.38,28.86$ and $26.96 \mathrm{ton} / \mathrm{ha}$, respectively. Raidor and ARC were the least productive cultivars, with means of 15.40 and 16.27 ton $\mathrm{DF} / \mathrm{ha}$, respectively. Forage production per cutting was inversely related to rainfall, highest yields were obtained with irrigation during the dry season. Mean crude protein, phosphorus, potassium, calcium and magnesium contents for all cuttings were 20.2, 0.40 , $4.54,1.39$ and $0.38 \%$, respectively. Results confirm that it is feasible to grow alfalfa for hay on well drained Cumulic Haplustolls in the southern coast with well adapted cultivars and proper agronomic practices.
\end{abstract}

\section{INTRODUCTION}

The dairy industry in Puerto Rico depends on concentrate feeds because the forage quality of tropical grasses and legumes is not sufficient to meet the nutritional requirements of specialized dairy cattle during. most of the lactation period (10). Alfalfa (Medicago sativa) is the highest quality forage used in intensive dairy production systems in the United States (11). It is fairly well adapted to the cool climate at high altitudes in the tropics of South America (4) and grows well on fertile soils in semiarid environments in the subtropics (9). However, it is very susceptible to foliar diseases in wet environments (3) and it does not tolerate acid soils high in soluble $\mathrm{Al}$ and $\mathrm{Mn}$ (7).

In Puerto Rico, previous research has indicated that with supplemental irrigation, alfalfa can be grown for hay in the semiarid southern region (5). Crop persistence has been affected by a fungus disease caused by Phytophthora megasperma under poorly drained conditions in the Lajas Valley (12). However, several cultivars were highly productive and persisted for 2 years in the semiarid climate and in the well drained soils of the southern coastal areas at Fortuna, Puerto Rico, with proper crop management (13)

'Manuscript submitted to Editorial Board, 16 July 1987.

${ }^{2}$ Associate Professor, Agronomy and Soils Department; Associate Agronomist, (ret.), Corozal Substation; and Research Assistant, Corozal Substation, respectively, Agricultural Experiment Station, Mayagüez Campus, University of Puerto Rico, Río Piedras, P. R. 
The objective of the present research was to continue studies on forage production and persistance with clipping at regular intervals of adapted alfalfa cultivars grown in a promising semiarid environment.

\section{MATERIALS AND METHODS}

The experiment was conducted at the Fortuna Agricultural Research and Development Center, lat. $18^{\circ} 2^{\prime} \mathrm{N}$ and long. $66^{\circ} 31^{\prime} \mathrm{W}$, in the semiarid region of Puerto Rico. Mean annual rainfall is $877 \mathrm{~mm}$ distributed mainly from May through November, and mean daily air temperature is $26 \mathrm{C}^{\circ}$. The soil is a San Antón clay (Cumulic Haplustolls), average $\mathrm{pH}$ is 7.5 with $48 \mathrm{p} / \mathrm{m}$ available $\mathrm{P}$ (Bray I) and $796 \mathrm{p} / \mathrm{m}$ exchangeable $\mathrm{K}$ in the top $20 \mathrm{~cm}$. A randomized complete block design with four replications was used. The treatments were nine alfalfa (Medicago sativa)] cultivars and a 1:1 mixture of two of the latter (both from Florida), planted January 1983. The alfalfa cultivars are identified in table 1 . The plots were $6.1 \times 1.8 \mathrm{~m}$ including 10 rows of plants with $25.5 \mathrm{~cm}$ between rows.

The seeds were inoculated with proper Rhizobia immediately before planting (2) and handseeded at a rate of $13.4 \mathrm{~kg} / \mathrm{ha}$. The herbicide Trifluralin was used as a preemergent at a rate of $1.4 \mathrm{~kg} \mathrm{a.i./ha}$ and incorporated into the soil $24 \mathrm{~h}$ prior to planting (1). The experiment was topdressed annually with $224 \mathrm{~kg} / \mathrm{ha}$ of $\mathrm{P}, 672 \mathrm{~kg} / \mathrm{ha}$ of $\mathrm{K}$ and $6 \mathrm{~kg} / \mathrm{ha}$ of borax. Aerial irrigations were applied daily after planting, and thereafter 3 to 5 times per month throughout the experimental period, except during the rainy months of September, October and November.

The plants were cut at a height of $5 \mathrm{~cm}$ in nearly full-bloom stage every 30 to 35 days. The green forage was weighed in the field and subsamples were dried at $60^{\circ} \mathrm{C}$ in a forced-air oven for $48 \mathrm{~h}$ for dry matter determination. Subsamples were composited by replication in each harvest interval and analyzed for $\mathrm{N}, \mathrm{P}, \mathrm{K}, \mathrm{Ca}$ and $\mathrm{Mg}$. Crude protein (CP) content was computed as $\mathrm{N} \times 6.25$.

TABLE 1.-Identification and origin of alfalfa cultivars

\begin{tabular}{|c|c|c|}
\hline & Cultivar & Origin \\
\hline 1. & Valador & Northrup King \& Co., Minneapolis, Minnesota \\
\hline 2. & Maxidor & Northrup King \& Co., Minneapolis, Minnesota \\
\hline 3 & Raidor & Northrup King \& Co., Minneapolis, Minnesota \\
\hline 4. & Brand 819 & Northrup King \& Co., Minneapolis, Minnesota \\
\hline 5. & $\mathrm{ARC}$ & $\begin{array}{l}\text { USDA-Agrieultural Research Service, } \\
\text { Beltsville, Maryland }\end{array}$ \\
\hline 6. & Moapa & $\begin{array}{l}\text { FAO-United Nations Development Program, } \\
\text { Santo Domingo, Dominican Republic }\end{array}$ \\
\hline 7. & Maracay & $\begin{array}{l}\text { Dept. Agronomy, Venezuela Central Univ., } \\
\text { Maracay, Venezuela }\end{array}$ \\
\hline 8. & Florida 66 & Agronomy Dept., Univ. of Florida, Gainesville \\
\hline 9. & Florida 77 & Agronomy Dept., Univ, of Florida, Gainesville \\
\hline
\end{tabular}


TABLE 2.-Mean dry forage yields of alfalfa cultivars at Fortuna during 1983

\begin{tabular}{|c|c|c|c|c|c|c|c|c|c|c|c|}
\hline \multirow[b]{2}{*}{ Cultivar } & \multicolumn{10}{|c|}{ Number and date of cutting } & \multirow[b]{2}{*}{ Mean } \\
\hline & $\begin{array}{c}1 \\
\operatorname{Mar} 29\end{array}$ & $\begin{array}{c}2 \\
\text { May } 2\end{array}$ & $\begin{array}{c}3 \\
\text { May } 31\end{array}$ & $\begin{array}{c}4 \\
\operatorname{Jun} 30\end{array}$ & $\begin{array}{c}5 \\
\text { Aug } 10\end{array}$ & $\begin{array}{c}6 \\
\text { Aug } 30\end{array}$ & $\begin{array}{c}7 \\
\text { Sept } 29\end{array}$ & $\begin{array}{c}8 \\
\text { Oct } 31\end{array}$ & $\begin{array}{c}9 \\
\operatorname{Dec} 5\end{array}$ & $\begin{array}{c}10 \\
\operatorname{Jan} 8\end{array}$ & \\
\hline \multicolumn{12}{|c|}{ tha } \\
\hline Florida 77 & $3.14^{1}$ & $3.33 \mathrm{a}$ & $2.92 \mathrm{a}$ & $3.63 \mathrm{a}$ & $2.36 \mathrm{a}$ & $2.05 \mathrm{abc}$ & $3.14 \mathrm{a}$ & $3.09 \mathrm{a}$ & $2.49 \mathrm{a}$ & $2.91 \mathrm{a}$ & $2.90 \mathrm{ab}$ \\
\hline Raidor & $3.04 \mathrm{a}$ & $3.21 \mathrm{a}$ & $2.42 \mathrm{a}$ & $2.78 \mathrm{a}$ & $1.78 \mathrm{a}$ & $1.63 \mathrm{bc}$ & $1.98 \mathrm{c}$ & $1.89 \mathrm{c}$ & $1.76 \mathrm{c}$ & $2.29 \mathrm{ab}$ & $2.28 \mathrm{abc}$ \\
\hline Brand & $2.79 a$ & $2.81 \mathrm{a}$ & $2.83 \mathrm{a}$ & $3.41 \mathrm{a}$ & $2.51 \mathrm{a}$ & $2.38 a b c$ & $3.23 \mathrm{a}$ & $3.18 \mathrm{a}$ & $2.15 \mathrm{abc}$ & $2.64 \mathrm{ab}$ & 2.84 abc \\
\hline Valador & $2.77 \mathrm{a}$ & $3.18 \mathrm{a}$ & $3.03 \mathrm{a}$ & $3.36 \mathrm{a}$ & $2.79 \mathrm{a}$ & $2.05 \mathrm{abc}$ & $3.24 \mathrm{a}$ & $3.03 \mathrm{a}$ & $2.32 \mathrm{abc}$ & $2.60 \mathrm{ab}$ & $2.91 \mathrm{a}$ \\
\hline Maracay & $2.68 \mathrm{a}$ & $3.37 \mathrm{a}$ & $3.12 \mathrm{a}$ & $3.52 \mathrm{a}$ & $2.70 \mathrm{a}$ & $2.50 \mathrm{a}$ & $3.24 \mathrm{a}$ & $3.03 \mathrm{a}$ & $2.32 \mathrm{abc}$ & $2.60 \mathrm{ab}$ & $2.91 \mathrm{a}$ \\
\hline Florida 66 & $2.57 \mathrm{a}$ & $2.76 \mathrm{a}$ & $2.59 \mathrm{a}$ & $3.56 \mathrm{a}$ & $2.88 \mathrm{a}$ & $2.16 \mathrm{abc}$ & $2.83 \mathrm{ab}$ & $2.95 \mathrm{a}$ & $2.34 \mathrm{ab}$ & $2.46 \mathrm{ab}$ & $2.72 \mathrm{abc}$ \\
\hline Florida $66 \& 77$ & $2.53 \mathrm{a}$ & $3.12 \mathrm{a}$ & $2.74 \mathrm{a}$ & $3.29 \mathrm{a}$ & $2.62 \mathrm{a}$ & $2.41 \mathrm{ab}$ & $3.16 \mathrm{a}$ & $2.97 \mathrm{a}$ & $2.31 \mathrm{abc}$ & $2.84 \mathrm{ab}$ & $2.80 \mathrm{abc}$ \\
\hline $\mathrm{ARC}$ & $2.48 \mathrm{a}$ & $3.40 \mathrm{a}$ & $2.28 \mathrm{a}$ & $2.57 \mathrm{a}$ & $1.72 \mathrm{a}$ & $1.44 \mathrm{c}$ & $2.10 \mathrm{be}$ & $2.07 \mathrm{c}$ & $1.83 \mathrm{bc}$ & $2.02 \mathrm{~b}$ & $2.20 \mathrm{c}$ \\
\hline Maxidor & $2.46 \mathrm{a}$ & $3.10 \mathrm{a}$ & $2.57 \mathrm{a}$ & $3.18 \mathrm{a}$ & $1.83 \mathrm{a}$ & $1.86 \mathrm{abc}$ & 2.47 abc & $2.31 b c$ & $1.79 b c$ & $1.79 \mathrm{bc}$ & $2.38 \mathrm{abc}$ \\
\hline Moapa & $2.28 \mathrm{a}$ & $2.98 \mathrm{a}$ & $2.97 \mathrm{a}$ & $3.09 \mathrm{a}$ & $2.51 \mathrm{a}$ & $1.94 \mathrm{abc}$ & $2.84 \mathrm{ab}$ & $2.64 \mathrm{ab}$ & $1.93 b c$ & $2.46 \mathrm{ab}$ & $2.56 \mathrm{abc}$ \\
\hline Mean & 2.70 & 3.13 & 2.75 & 3.24 & 2.37 & 2.04 & 2.79 & 2.70 & 2.11 & 2.49 & 2.63 \\
\hline
\end{tabular}

'Means in colums followed by the same letter do not differ significantly at the $5 \%$ probability level, Duncan's Multiple Range Test. 
Duncan's Multiple Range Test was used to compare treatments with regard to dry forage (DF) yield for each harvest, for each year and over the full 3-year experiment.

\section{RESULTS AND DISCUSSION}

All cultivars were highly productive in 1983 , the first year of the experiment, with mean annual yields of over $2.2 \mathrm{t} \mathrm{DF} / \mathrm{ha}$ per cutting (table 2). The most productive cultivar, Maracay, was not significantly different from the others $(\mathrm{P}=0.05)$ among cultivars for the first five cuttings; thus all were fairly well adapted to environmental conditions at Fortuna. Yields of cultivars ARC and Raidor began to decline in the rainy season, August through November, but they recovered after the December cutting.

During the second year of the experiment, most of the cultivars, other than ARC and Raidor, were highly productive with mean annual yields over $2.5 \mathrm{t} \mathrm{DF} / \mathrm{ha}$ per cutting (table 3). Florida 77 produced the highest mean yield, but it was significantly higher than ARC and Raidor only. All cultivars produced high yields of DF in most of the cuttings. $\mathrm{DF}$ yields in 1984 were greater than in 1983, except during the rainy season, October through December.

The mean annual yields of the Florida cultivars were over $2.0 \mathrm{t} \mathrm{DF} / \mathrm{ha}$ per cutting during the third year of the experiment (table 4). Florida 77 was the most productive cultivar, although it was not significantly different $(\mathrm{P}=0.05)$ from Florida 66 and the mixture of these two. Maracay, Valador, Brand 819, Maxidor and Moapa were intermediate; ARC and Raidor produced the lowest yields and were invaded by weeds toward the end of the experiment.

Florida 77 was the most productive cultivar during the three years of the experiment with a mean total yield of $28.87 \mathrm{t} \mathrm{DF} / \mathrm{ha} / \mathrm{year}$, although this advantage was not significant $(P=0.05)$, except when compared with Raidor and ARC, which produced the lowest yields (table 5). The mean total DF production for all cultivars in 1983 and 1984, 26.31 and $27.16 \mathrm{t}$ $\mathrm{DF} / \mathrm{ha} / \mathrm{year}$, respectively, were not significantly different $(\mathrm{P}=0.05)$. Thereafter, 1985 yields declined significantly $(\mathrm{P}=0.05)$ to $15.77 \mathrm{t} \mathrm{DF} / \mathrm{ha} /$ year (table 5).

Forage productivity was related to rainfall distribution for most of the duration of the experiment. Highest yields were usually obtained with irrigation in the drier months of the year, whereas heavy rainfall during April and May, and September, October and November resulted in lower yields per cutting (fig. 1). Apparently, alfalfa cultivars were tolerant to a root rot caused by Phytophtora megasperma, but were still susceptible to temporary waterlogged conditions right after intensive rainfall in the rainy season. 
TABLE 3.-Mean dry forage yields of alfalfa cultivars at Fortuna during 1984

\begin{tabular}{|c|c|c|c|c|c|c|c|c|c|c|c|}
\hline \multirow[b]{2}{*}{ Cultivar } & \multicolumn{10}{|c|}{ Number and date of eutting } & \multirow[b]{2}{*}{ Mean } \\
\hline & $\begin{array}{c}11 \\
\text { Feb } 16\end{array}$ & $\begin{array}{c}12 \\
\operatorname{Mar} 21\end{array}$ & $\begin{array}{c}13 \\
\text { Apr } 23\end{array}$ & $\begin{array}{c}14 \\
\text { May } 21\end{array}$ & $\begin{array}{c}15 \\
\operatorname{Jun} 25\end{array}$ & $\begin{array}{c}16 \\
\text { Aug } 2\end{array}$ & $\begin{array}{c}17 \\
\text { Sept } 4\end{array}$ & $\begin{array}{c}18 \\
\text { Oct } 5\end{array}$ & $\begin{array}{c}19 \\
\text { Nov } 9\end{array}$ & $\begin{array}{c}20 \\
\text { Dec } 13\end{array}$ & \\
\hline \multicolumn{12}{|c|}{ t/ha } \\
\hline Florida 77 & $3.13 \mathrm{a}^{1}$ & $4.29 \mathrm{a}$ & $4.13 \mathrm{a}$ & $3.40 \mathrm{a}$ & $3.84 \mathrm{ab}$ & $3.55 \mathrm{a}$ & $3.56 \mathrm{ab}$ & $2.78 \mathrm{a}$ & $1.51 \mathrm{ab}$ & $1.90 \mathrm{ab}$ & $3.22 \mathrm{a}$ \\
\hline Maracay & $3.11 \mathrm{a}$ & $3.76 \mathrm{ab}$ & $3.93 \mathrm{a}$ & $3.30 \mathrm{a}$ & $4.01 \mathrm{a}$ & $3.43 \mathrm{ab}$ & $3.77 \mathrm{a}$ & $2.22 \mathrm{bc}$ & $1.42 \mathrm{ab}$ & $1.88 \mathrm{ab}$ & $3.08 \mathrm{a}$ \\
\hline Valador & $2.96 \mathrm{a}$ & $3.96 \mathrm{a}$ & $4.10 \mathrm{a}$ & $3.27 \mathrm{a}$ & $4.08 \mathrm{a}$ & $2.95 \mathrm{abc}$ & $3.44 \mathrm{abc}$ & $2.14 \mathrm{bcd}$ & $1.49 \mathrm{ab}$ & $1.77 \mathrm{ab}$ & $3.02 \mathrm{a}$ \\
\hline Florida $66 \& 77$ & $2.94 \mathrm{a}$ & $3.41 \mathrm{ab}$ & $3.51 \mathrm{a}$ & $3.13 \mathrm{ab}$ & $3.68 \mathrm{ab}$ & $3.11 \mathrm{abc}$ & $3.30 \mathrm{abc}$ & $2.44 \mathrm{ab}$ & $1.70 \mathrm{a}$ & $1.94 \mathrm{ab}$ & $2.91 \mathrm{a}$ \\
\hline Florida 66 & $2.85 \mathrm{a}$ & $3.55 \mathrm{ab}$ & $3.59 \mathrm{a}$ & $3.03 \mathrm{abc}$ & $3.19 a b$ & $3.15 \mathrm{abc}$ & $3.02 \mathrm{abc}$ & $2.51 \mathrm{ab}$ & $1.50 \mathrm{ab}$ & $2.03 \mathrm{a}$ & $2.84 \mathrm{ab}$ \\
\hline Moapa & $2.54 \mathrm{a}$ & $3.27 \mathrm{ab}$ & $3.69 \mathrm{a}$ & $2.81 \mathrm{abc}$ & $2.92 \mathrm{~b}$ & $2.81 \mathrm{abc}$ & $2.89 \mathrm{abc}$ & $1.82 \mathrm{~d}$ & $1.11 \mathrm{ab}$ & $1.40 \mathrm{~b}$ & $2.53 \mathrm{abc}$ \\
\hline Brand 819 & $2.54 \mathrm{a}$ & $3.66 \mathrm{ab}$ & $3.75 \mathrm{a}$ & $3.07 \mathrm{abc}$ & $3.47 \mathrm{ab}$ & $3.00 \mathrm{abc}$ & $3.21 \mathrm{abc}$ & $1.88 \mathrm{~cd}$ & $1.24 \mathrm{ab}$ & $1.59 \mathrm{ab}$ & $2.74 \mathrm{ab}$ \\
\hline Maxidor & $2.51 \mathrm{a}$ & $3.28 \mathrm{ab}$ & $3.48 \mathrm{a}$ & $2.53 \mathrm{bc}$ & $3.53 \mathrm{ab}$ & $2.85 \mathrm{abc}$ & $3.44 \mathrm{abc}$ & $1.91 \mathrm{bed}$ & $1.00 \mathrm{~b}$ & $1.55 \mathrm{ab}$ & $2.61 a b c$ \\
\hline Raidor & $2.46 \mathrm{a}$ & $2.82 \mathrm{~b}$ & $3.11 \mathrm{a}$ & $2.42 \mathrm{c}$ & $3.18 \mathrm{ab}$ & $2.38 \mathrm{abc}$ & $2.34 \mathrm{c}$ & $0.95 \mathrm{w}$ & $0.34 \mathrm{c}$ & $0.39 \mathrm{c}$ & $2.04 \mathrm{c}$ \\
\hline ARC & $2.35 \mathrm{a}$ & $2.87 \mathrm{~b}$ & $3.34 a$ & $2.49 \mathrm{bc}$ & $3.04 \mathrm{ab}$ & $2.62 \mathrm{bc}$ & $2.51 \mathrm{bc}$ & $1.22 \mathrm{e}$ & $0.90 \mathrm{~b}$ & $0.48 \mathrm{c}$ & $2.18 \mathrm{bc}$ \\
\hline Mean & 2.74 & 3.49 & 3.66 & 2.94 & 3.49 & 2.98 & 3.15 & 1.99 & 1.22 & 1.50 & 2.72 \\
\hline
\end{tabular}

'Means in columns followed by the same letter do not differ significantly at the 5\% probability level, Duncan's Multiple Range Test. 
TABLE 4.-Mean dry forage yields of alfalfa cultivars at Fortuna during 1985

\begin{tabular}{|c|c|c|c|c|c|c|c|c|c|c|c|}
\hline \multirow[b]{2}{*}{ Cultivar } & \multicolumn{10}{|c|}{ Number and date of cutting } & \multirow[b]{2}{*}{ Mean } \\
\hline & $\begin{array}{c}21 \\
\operatorname{Jan} 18\end{array}$ & $\begin{array}{c}22 \\
\text { Feb } 25\end{array}$ & $\begin{array}{c}23 \\
\operatorname{Mar} 27\end{array}$ & $\begin{array}{c}24 \\
\text { Apr } 29\end{array}$ & $\begin{array}{c}25 \\
\text { May } 30\end{array}$ & $\begin{array}{c}26 \\
\operatorname{Jun} 28\end{array}$ & $\begin{array}{c}27 \\
\text { Aug } 2\end{array}$ & $\begin{array}{c}28 \\
\text { Sept } 4\end{array}$ & $\begin{array}{c}29 \\
\text { Oet } 10\end{array}$ & $\begin{array}{c}30 \\
\text { Nov } 20\end{array}$ & \\
\hline \multicolumn{12}{|c|}{ t/ha } \\
\hline Florida 77 & $2.69 \mathrm{a}^{2}$ & $2.83 \mathrm{a}$ & $3.07 \mathrm{a}$ & $3.92 \mathrm{a}$ & $3.26 a$ & $3.36 \mathrm{a}$ & $2.75 \mathrm{a}$ & $1.66 \mathrm{a}$ & $0.87 \mathrm{a}$ & 0.96 & $2.54 \mathrm{a}$ \\
\hline Florida $66 \& 77$ & $2.32 \mathrm{ab}$ & $2.75 \mathrm{a}$ & $2.90 \mathrm{ab}$ & $3.15 \mathrm{ab}$ & $3.07 \mathrm{ab}$ & $2.88 \mathrm{ab}$ & $2.89 \mathrm{a}$ & $1.68 \mathrm{a}$ & $0.98 \mathrm{a}$ & $1.08 \mathrm{a}$ & $2.37 \mathrm{ab}$ \\
\hline Maracay & $2.25 \mathrm{abc}$ & $2.62 \mathrm{ab}$ & $2.82 \mathrm{ab}$ & $3.04 \mathrm{ab}$ & $2.50 a b c$ & $2.25 \mathrm{bc}$ & $1.63 b c$ & $0.55 \mathrm{bcd}$ & $0.38 b c$ & $0.22 \mathrm{~b}$ & $1.83 \mathrm{bc}$ \\
\hline Valador & 2.24 abc & $2.44 \mathrm{ab}$ & $2.78 \mathrm{ab}$ & $2.98 \mathrm{ab}$ & $2.40 \mathrm{bc}$ & $2.44 \mathrm{ab}$ & $1.54 \mathrm{bc}$ & $0.73 \mathrm{bc}$ & 0.32 bed & $0.25 \mathrm{~b}$ & $1.81 b c$ \\
\hline Florida 66 & $2.20 \mathrm{abc}$ & $2.33 a b c$ & $2.80 \mathrm{ab}$ & $3.05 \mathrm{ab}$ & $2.64 \mathrm{abc}$ & $2.68 \mathrm{ab}$ & $2.20 \mathrm{abc}$ & $1.13 \mathrm{ab}$ & $0.72 \mathrm{a}$ & $0.77 \mathrm{a}$ & $2.05 \mathrm{abc}$ \\
\hline Brand 819 & $1.78 \mathrm{bc}$ & $2.11 \mathrm{bc}$ & $2.34 \mathrm{bc}$ & $2.87 \mathrm{~b}$ & $2.47 \mathrm{abc}$ & $2.32 \mathrm{~b}$ & $1.50 \mathrm{bec}$ & $0.80 \mathrm{bc}$ & $0.40 \mathrm{~b}$ & $0.31 \mathrm{~b}$ & $1.69 \mathrm{~cd}$ \\
\hline Moapa & $1.68 \mathrm{bc}$ & $1.80 \mathrm{c}$ & $1.90 \mathrm{c}$ & $2.25 \mathrm{~b}$ & $1.86 \mathrm{c}$ & $1.38 \mathrm{c}$ & $0.66 \mathrm{~cd}$ & $0.19 \mathrm{~cd}$ & $0.08 \mathrm{bcd}$ & $0.01 \mathrm{~b}$ & $1.18 \mathrm{~d}$ \\
\hline Maxidor & $1.53 \mathrm{c}$ & $2.16 \mathrm{bc}$ & $2.51 \mathrm{ab}$ & $2.47 \mathrm{~b}$ & $2.10 \mathrm{c}$ & $2.20 \mathrm{bc}$ & $1.16 \mathrm{bc}$ & $0.39 \mathrm{~cd}$ & $0.17 \mathrm{bed}$ & $0.18 \mathrm{~b}$ & $1.49 \mathrm{~cd}$ \\
\hline ARC & $0.69 \mathrm{~d}$ & $0.94 \mathrm{~d}$ & $0.99 \mathrm{~d}$ & $1.15 \mathrm{c}$ & $0.76 \mathrm{~d}$ & $0.44 \mathrm{~d}$ & $0.10 \mathrm{~d}$ & $0.03 \mathrm{~d}$ & $0.05 \mathrm{~cd}$ & $0.00 \mathrm{c}$ & $0.51 \mathrm{e}$ \\
\hline Raidor & $0.53 \mathrm{~d}$ & $0.46 \mathrm{~d}$ & $0.61 \mathrm{~d}$ & $0.69 \mathrm{c}$ & $0.39 \mathrm{~d}$ & $0.27 \mathrm{~d}$ & $0.08 \mathrm{~d}$ & $0.02 \mathrm{~d}$ & $0.00 \mathrm{e}$ & $0.00 \mathrm{c}$ & $0.30 \mathrm{e}$ \\
\hline Mean & 1.79 & 2.04 & 2.27 & 2.56 & 2.15 & 2.02 & 1.45 & 0.72 & 0.40 & 0.38 & 1.58 \\
\hline
\end{tabular}

${ }^{1}$ Means in columns followed by the same letter do not differ significantly at the $5 \%$ probability level, Duncan's Multiple Range Test. 
TABLE 5.-Mean total dry forage yields of alfalfa cultivars at Fortuna from 1983 to 1985

\begin{tabular}{|c|c|c|c|c|}
\hline \multirow[b]{2}{*}{ Cultivar } & \multicolumn{3}{|c|}{ Year } & \multirow[b]{2}{*}{ Mean } \\
\hline & 1983 & 1984 & 1985 & \\
\hline \multicolumn{5}{|c|}{ tha } \\
\hline Maracay & $29.09 \mathrm{a}^{1}$ & $30.81 \mathrm{a}$ & $18.26 \mathrm{bc}$ & $26.05 \mathrm{ab}$ \\
\hline Florida 77 & $29.05 \mathrm{a}$ & $32.19 \mathrm{a}$ & $25.36 \mathrm{a}$ & $28.86 \mathrm{a}$ \\
\hline Valador & $28.38 \mathrm{abc}$ & $30.16 \mathrm{a}$ & $18.11 b c$ & $25.55 \mathrm{abc}$ \\
\hline Florida $66 \& 77$ & $28.04 a b c$ & $29.14 \mathrm{a}$ & $23.70 \mathrm{ab}$ & $26.96 \mathrm{ab}$ \\
\hline Florida 66 & $27.21 \mathrm{abc}$ & $28.42 \mathrm{ab}$ & $20.52 \mathrm{abc}$ & $25.38 \mathrm{abc}$ \\
\hline Brand 819 & $27.18 \mathrm{abc}$ & $27.41 \mathrm{ab}$ & $16.91 \mathrm{~cd}$ & $23.83 \mathrm{bc}$ \\
\hline Moapa & $25.63 \mathrm{abc}$ & $25.28 \mathrm{abc}$ & $11.81 \mathrm{~d}$ & $20.90 \mathrm{bed}$ \\
\hline Maxidor & $23.79 \mathrm{abc}$ & $26.06 \mathrm{abc}$ & $14.89 \mathrm{ed}$ & 21.58 be \\
\hline Raidor & $22.77 \mathrm{abc}$ & $20.40 c$ & $3.04 \mathrm{e}$ & $15.40 \mathrm{~d}$ \\
\hline ARC & $21.96 \mathrm{c}$ & $21.81 \mathrm{bc}$ & $5.15 \mathrm{e}$ & $16.27 \mathrm{~d}$ \\
\hline Mean & 26.31 & 27.17 & 15.77 & 23.08 \\
\hline
\end{tabular}

${ }^{1}$ Means in columns followed by the same letter do not differ significantly at the $5 \%$ probability level, Dunean's Multiple Range Test.

Yields of DF of the well-adapted alfalfa cultivars Florida 66 and 77, and the mixture of these two cultivars, were higher in the present investigation than those reported in Florida (8) and the Dominican Republic (6), but similar results were obtained in a previous experiment conducted in the same region of Puerto Rico in the first year of evaluation (13). However, crop persistance in this experiment was better; acceptable yields were still produced after 27 cuttings (table 4 ).

Crude protein, $\mathrm{P}, \mathrm{K}, \mathrm{Ca}$ and $\mathrm{Mg}$ content, means for all cuttings, were $20.2,0.40,4.54, .1 .39$, and $0.38 \%$, respectively (table 6 ). These values are similar to those reported before in a similar experiment in the same location (13), except for $K$, which was about $1 \%$ higher in the present trial.

These results confirm that it is feasible to grow alfalfa for hay with adapted cultivars on the well drained Cumulic Haplustolls soils in the semiarid region of Puerto Rico. Proper agronomic practices, including inoculation with effective Rhizobia, fertilization with $\mathrm{P}$ and $\mathrm{K}$, weed and insect control when required, and supplemental irrigation during the dry season assure good yields and crop persistence.

\section{RESUMEN}

Producción de forraje de nueve cultivares de alfalfa en la región semiárida de Puerto Rico

Se realizó un experimento por tres años para determinar la producción de forraje seco (DF) en el Centro de Investigación y Desarrollo de Fortuna en la costa sur de Puerto Rico de 1983 a 1985. Las cultivares más productivas al cabo de los tres años fueron Florida 66 y 77 y una mezcla de ambas, con una producción media anual de $25.38,28.86$ y 26.96 tons. de DF/ha., 


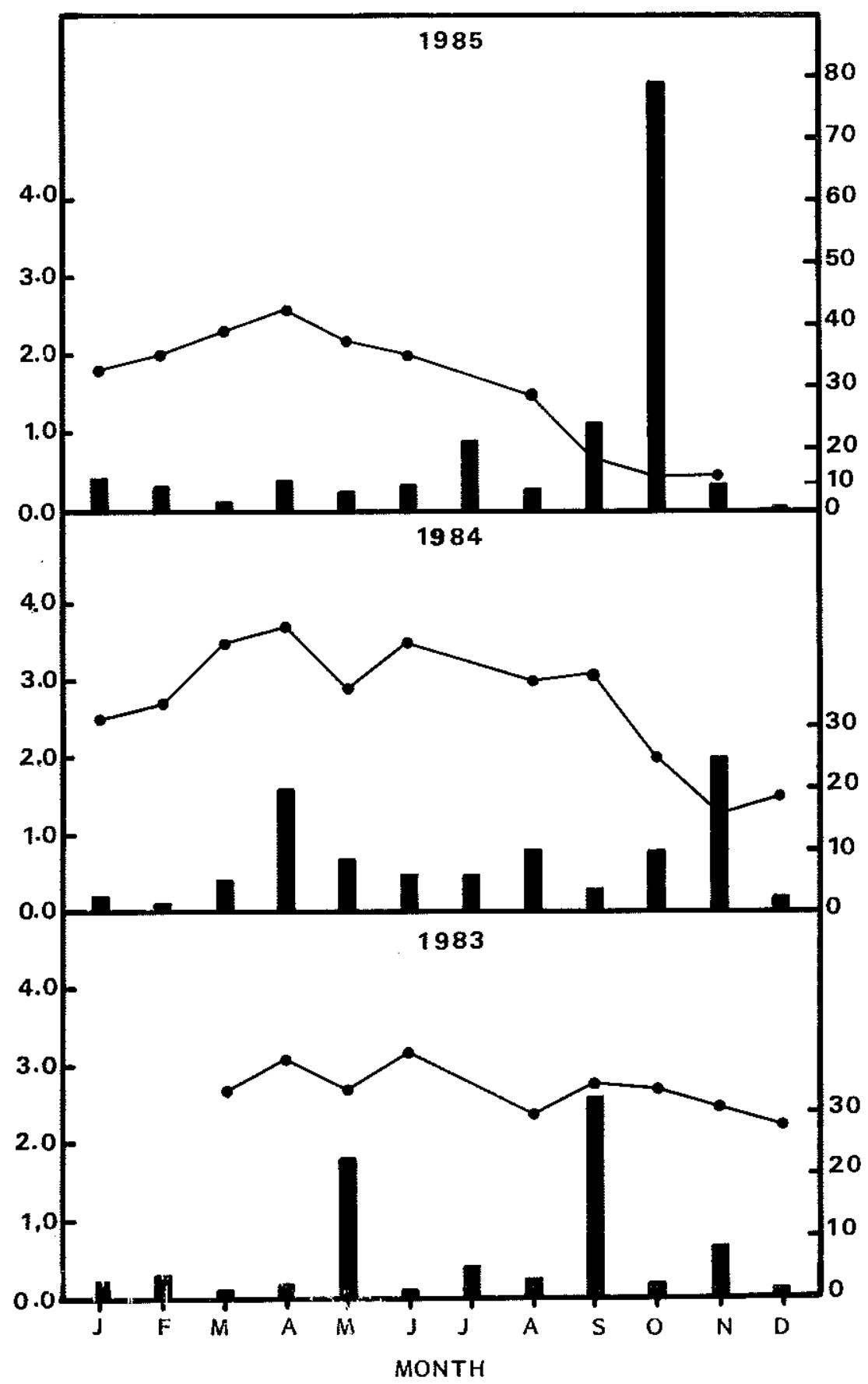

FIG. 1.-Seasonal dry mean forage yields for all alfalfa cultivars eut every 28 to 38 days. 
TABLE 6.-Crude protein and mineral contents ${ }^{2}$ of alfalfa cultivars at Fortuna from 1983 to 1985

\begin{tabular}{|c|c|c|c|c|c|}
\hline Cultivar & Crude protein & $\mathrm{P}$ & $\mathrm{K}$ & $\mathrm{Ca}$ & $\mathrm{Mg}$ \\
\hline \multicolumn{6}{|c|}{$\%$} \\
\hline Florida 77 & 20.9 & 0.42 & 4.51 & 1.45 & 0.39 \\
\hline Florida $66 \& 77$ & 21.3 & 0.42 & 4.60 & 1.41 & 0.39 \\
\hline Maracay & 17.9 & 0.37 & 4.21 & 1.40 & 0.35 \\
\hline Valador & 20.3 & 0.40 & 4.67 & 1.43 & 0.38 \\
\hline Florida 66 & 18.7 & 0.39 & 4.33 & 1.42 & 0.37 \\
\hline Brand $81 \$ 9$ & 19.9 & 0.39 & 4.52 & 1.41 & 0.37 \\
\hline Moapa & 19.8 & 0.40 & 4.62 & 1.40 & 0.36 \\
\hline Maxidor & 19.6 & 0.39 & 4.56 & 1.43 & 0.38 \\
\hline ARC & 21.1 & 0.40 & 4.60 & 1.28 & 0.40 \\
\hline Raidor & 22.9 & 0.43 & 4.75 & 1.29 & 0.38 \\
\hline Mean & 20.2 & 0.40 & 4.54 & 1.39 & 0.38 \\
\hline
\end{tabular}

${ }^{1}$ Mean for all cuttings.

respectivamente. La producción por corte estuvo relacionada con la distribución de las lluvias, siendo menor durante los meses más lluviosos, debido posiblemente a las condiciones ocasionales de encharcamiento a pesar de que el suelo tiene características de buen drenaje. El contenido medio en todos los cortes en proteína bruta, fósforo, potasio, calcio y magnesio de las 9 cultivares fue $20.2,0.40,4.54,1.39$ y 0.38 , respecivamente, el cual fue similar a otros resultados obtenidos en un experimento anterior en la misma región. Los resultados confirman que es posible sembrar alfalfa para heno en suelos Cumulic Haplustolls bien desaguados en la región semiárida, contando con cultivares adaptadas e irrigación, y realizando prácticas agronómicas adecuadas.

\section{LITERATURE CITED}

1. Almodóvar-Vega, L. and J. Vélez-Santiago, 1980. Evaluation of preplant incorporated chemicals for weed control on alfalfa in a Vertisol, J. Agric. Univ. P. R. 64 (1): $129-30$.

2. Anonymous, 1975. Pelleting small legume seeds with Pelinoe, Information Bull, 1622, Nitragin Co. Res. Dep.

3. Buritica, C. P. y G. E. Ponce, 1978. Control de enfermedades foliares de la alfalfa (Medicago sativa L.) en el departamento de Narin̄o. Pasto, Instituto Colombiano Agropecuario.

4. Crowder, L. V., 1967. Grasslands of Colombia, Herb. Abstr. 37 (4) : 237-45.

5. Freyre, R. H., M. Rico-Ballester, R. Vázquez-Romero and H. E. Warmke, 1954. Alfalfa yields declined between the fifth and eighth harvest, USDA Exp. Stn. Ann. Prog. Rep.

6. Gutiérrez de Flores, T., F. Cuevas-Pérez, J. A. Arroyo-Aguilú, B. Wagner, R. A. Peterson and K. Santhirasegaram, 1977. Adaptación, comportamiento y producción de alfalfa (Medicago sativa) en la República Dominicana, Presentado en el 8vo. Congreso Panamericano de Medicina Veterinaria y Zootecnia, Santo Domingo, República Dominieana.

7. Helyar, K. R., 1978. Effects of aluminum and manganese toxicities on legume growth, in pp. 207-31 C. S. Andrew and E. J. Kamprath (Eds), Workshop on Mineral Nutrition of Legumes in Tropical and Subtropical Soils, Brisbane, Australia, CSIRO. 
8. Horner, E. S. and C. O., Ruelke, 1980. Florida 77 alfalfa and recommended management practices for its production, Agric. Exp. Stn. Univ. Florida, Gainesville, Fla. Circ. S-271.

9. Sprague, H. B., 1975. Major forage legumes for improved grazing lands and forage harvest in the tropics and subtropics, in pp. 59-76, Characteristics of economically important food and forage legumes and forage grasses for the tropics and subtropics, U. S. Agency for International Development, Agriculture Technology for Developing Countries, Technical Series Bull. 14.

10. Stobbs, T. H., 1976. Milk production per cow and per hectare from tropical pastures, (Milk production from tropical pastures), in pp. 129-46, Memoria Seminario Internacional de Ganadería Tropical, Acapulco, México, Secretaría de Agricultura y Ganadería.

11. Van Horn, H. H., 1986. Alfalfa "Florida 77" y maní perenne ([Florigraze rizoma): dops leguminosas para ganado lechero, Conferencia Internacional sobre Ganadería y Avicultura en los Trópicos, Centro de Agricultura Tropical, Univ. Florida, Gainesville, Fla.

12. Vélez-Santiago, J., J. A. Arroyo-Aguilú, S. Torres and N. Corchado-Juarbe, 1983. Performance and chemical composition of 18 nondormant alfalfa cultivars at the Lajas Valley, J. Agric. Univ. P. R. 67 (3): 204-12.

13.,,$-- F$. Fuentes and A. Torres, 1984. Evaluation of eight alfalfa cultivars in a Cumulic Haplustolls of southern Puerto Rico, J. Agric. Univ. P. R. 68 (2): 121-30. 